\title{
Biological activity and applications of pyocyanin produced by Pseudomonas aeruginosa
}

\author{
Diaa A Marrez ${ }^{1 *}$ and Haitham S Mohamad ${ }^{2}$ \\ ${ }^{1}$ Food Toxicology and Contaminants Department, National Research Centre, Egypt \\ ${ }^{2}$ Microbiology Department, National Research Centre, Egypt
}

\begin{abstract}
There is growing interest in microbial pigments due to their natural character, safe to use, medicinal properties and rich in nutrients like vitamins. Production of these pigments is independent of season and geographical condition. Moreover, microbial pigments can be produced from waste material reducing water and environmental pollutions. Pseudomonas aeruginosa produce a wide variety of pigments as secondary metabolites, which play an important role in interactions between Pseudomonas species and other organisms. Four major different pigments have been described in P. aeruginosa produce variety of redox-active phenazine compounds, including pyocyanin, fluorescein, pyorubrin and pyomelanin. Pyocyanin is a chloroform soluble blue green phenazine pigment produced by active cultures of $P$. aeruginosa. Pyocyanin has antibiotic activity against bacteria, fungi and protozoa. About 90 to $95 \%$ of P. aeruginosa strains produce pyocyanin which was the main phenazine pigment associated with organism and had powerful antimicrobial, antioxidant and anticancer activities.
\end{abstract}

KEYWORDS: Pyocyanin; Pseudomonas aeruginosa; Antimicrobial; Antioxidant; Anticancer

ABBREVIATIONS: SSV: Soyabean Stunt Virus; MFCs: Microbial fuel cells; ROS: Reactive Oxygen Species; SAM: S-adenosyl Methionine; PCA: Phenazine-1-carboxylic Acid

\section{PYOCYANIN}

Pyocyanin is a blue redox-active secondary metabolite and a member of the large family of the tricyclic compounds, phenazines. They are secreted at the late stationary phase and provide a characteristic blue color to the medium. Because of its solubility in chloroform it can be easily isolated from culture medium. Chemically, pyocyanin is 5-methyl-1-hydroxyphenazine and can undergo complex series of oxidation-reduction reaction [1]. The elucidation of the structure of pyocyanin represents the first reported instance of the occurrence of the phenazine nucleus as a natural product. Pyocyanin can exist in either oxidized or reduced form, the latter being an unstable form of pyocyanin that reacts rapidly with molecular oxygen [2].

\section{Biosynthesis of Pyocyanin}

Two steps have been suggested the synthesis of pyocyanin from phenazine-1-carboxylic acid (PCA), which is the common precursor for many different species-specific phenazines. There are two reaction strategies by which PCA can be converted to pyocyanin. The first, PCA is first acted upon by the enzyme PhzM, S-adenosyl methionine (SAM) dependent methyltransferase and gets converted to 5-methylphenazine-1-carboxylic acid betaine
Quick Response Code:

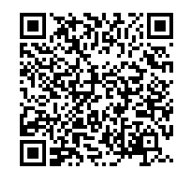

Address for correspondence: Diaa A Marrez, Food Toxicology and Contaminants Department, National Research Centre, Egypt

Received: December 20, 2019 Published: January 22, 2020

How to cite this article: Diaa A Marrez and Haitham SM. Biological activity and applications of pyocyanin produced by Pseudomonas aeruginosa. 2020 - 2(1) OAJBS.ID.000133.

DOI: $10.38125 / \mathrm{OAJBS} .000133$ 
by transfer of a methyl group to an $\mathrm{N}$ atom of the phenazine-ring moiety. This is followed by the action of the enzyme PhzS, a FADdependent monooxygenase, which involves the hydroxylative decarboxylation of 5-methylphenazine-1-carboxylic acid betaine to pyocyanin [3]. On the other hand, in the other reaction strategy leading to the biosynthesis of pyocyanin, PCA first gets converted to hydroxyphenazine in presence of the enzyme PhzS followed by its methylation to pyocyanin in presence of the SAM dependent methyltransferase, PhzM [4].

The synthesis of pyocyanin is affected by carbon and nitrogen sources in growth media, but most nutrients support pyocyanin production as long as the phosphate ion concentration is low and there is adequate sulfate ion present. Synthesis of this pigment also appears to be under the control of iron concentration since addition of iron to a medium containing low phosphate stimulates the synthesis of pyocyanin and related phenazine pigments by other species of bacteria [5].

\section{Mode of Action of Pyocyanin Against Target Cells}

The potent antagonistic action of pyocyanin was identified as the result of its unique redox potential by accepting a single electron, yielding a relatively stable anion radical and readily undergoing a redox cycle. During respiration, pyocyanin becomes reduced and unvaliantly reduces oxygen to the toxic superoxide radical. Accordingly, the antibiotic action of pyocyanin might be an expression of the toxicity of the $\mathrm{O}_{2}-$ and of $\mathrm{H}_{2} \mathrm{O}_{2}$ produced in increased amounts in its presence [6]. The resistance of various bacteria to pyocyanin would therefore be dependent upon the levels of superoxide dismutase and catalase possessed by the organism and on the presence of oxygen [7].

Hassan \& Fridovich [6] described a mechanism for the toxicity of pyocyanin whereby electron flow from biological pathways is diverted to increase the production of intracellular $\mathrm{O}_{2}$ reduction products, leading to cell death. It is of interest that $P$. aeruginosa, a "strict" aerobe, is itself insensitive to pyocyanin and seemingly escapes free-radical injury during production of or exposure to this compound [2]. Physiological studies have shown that P. aeruginosa resists the toxicity of this compound with increased superoxide dismutase and catalase activities under pyocyanin-producing conditions [8]. Baron \& Rowe [7] reported that the antimicrobial action of pyocyanin was bactericidal in nature and the effect was dependent on pyocyanin concentration.

\section{Biological Activity of Pyocyanin}

Pyocyanin is a good example of a secondary metabolite, which has antibiotic activities and able to co-ordinate the response of microbial communities to changes in the environment. The mechanism by which pyocyanin inhibits bacterial growth was investigated and it was concluded that, pyocyanin interacts with the cell membrane respiratory chain resulting in the inability of the bacterial cells to perform their active metabolic transport process $[8,9]$. Pyocyanin has variety of pharmacological effects on eukaryotic and prokaryotic cells [10] and, had antimicrobial activity against bacteria, fungi and protozoa [11]. The pyochelin and pyocyanin act synergistically to produce active oxygen species that cause cell damage and it subsequently leads to induced resistance [12].

\section{Antibacterial activity of Pyocyanin}

Saha et al. [13] reported that nearly $90-95 \%$ of antimicrobial inhibitions of $P$. aeruginosa strains were due to production of the water-soluble secondary metabolite pyocyanin. It showed antagonistic activity against pathogenic bacteria like Salmonella paratyphi, E. coli, Klebsiella pneumonia. Pyocyanin isolated from $P$. aeruginosa 4B strain showed antibiotic activities against various pathogens and food spoilage bacteria like Listeria monocytogens and Bacillus cereus. The secondary metabolite along with various enzymes like haemolysin and hydrolytic enzymes played a key role for their antimicrobial activities [14].

Rahman et al. [15] revealed that pyocyanin from $P$. aeruginosa DSO-129 has antimicrobial effect on organisms like S. aureus, Staphylococcus epidermis, Bacillus subtilis, Micrococcus luteus and Saccharomyces cerevisiae. There has been another report related to the antibiotic activity of pyocyanin against different pathogens. The pigment produced by the strain showed very effective activity against organisms like E. coli, Acinetobacter, S. aureus and Streptococcus pneumonia [16]. Pyocyanin from $P$. aeruginosa has antibacterial activity toward competing bacteria including indoleproducing E. coli [2]. Also, Sudhakar et al. [17] reported that pyocyanin from $P$. aeruginosa SU1against E. coli, S. aureus, Proteus sp., Klebsiella sp. and Pseudomonas sp. The maximum activity was seen against E. coli, S. aureus, Proteus sp., Klebsiella sp., whereas Pseudomonas sp. showed resistance.

\section{Antifungal Activity of Pyocyanin}

Pyocyanin inhibit the growth of various Candida spp. and Aspergillus fumigatus; pyocyanin may also inhibit yeast-mycelial transformation in C. albicans [18]. Pyocyanin induced triggering systemic resistance against Fusarium wilt of tomato [12]. Pyocyanin has antifungal activity against $A$. niger [18]. Pyocyanin isolated from P. aeruginosa also inhibited the growth of fungi like Aspergillus fumigatus and Candida albicans isolated from the sputum of CF patients [19]. There was a clinical evidence that pyocyanin suppressed the growth of different species of $C$. albicans in patients with lung infection. Reoccurring of $C$. albicans was noticed after the suppression of pyocyanin. Substances like pyocyanin, pyrrolnitrin and pseudomonic acid produced by $P$. aeruginosa showed antibiotic actions in vivo on Candida species grown on Sabroud's Dextrose Agar $[20,21]$. Signal mediated interactions between $P$. aeruginosa and $C$. albicans in CF patients was found. The presence of $\mathrm{N}$-acyl homoserine lactones (HSLS) produced by P. aeruginosa affected the morphology of $C$. albicans. In the same way $C$. albicans inhibited the swarming motility of $P$. aeruginosa. When $P$. aeruginosa was cocultured with $C$. albicans, the former synthesised large amounts of pyocyanin and even the growth of $C$. albicans was inhibited [2224]. Sudhakar et al. [17] reported the production of pyocyanin from P. aeruginosa WS1 and its antagonistic activity against commonly encountered phytopathogens. The MIC of pyocyanin was further analysed against phytopathogens and was found to be $64 \mathrm{\mu g} \mathrm{ml}^{-1}$ against Aspergillus flavus and Aspergillus fumigates, and $128 \mu \mathrm{g} \mathrm{ml}^{-1}$ against Candida species.

\section{Antioxidant Activity of Pyocyanin}

Rada et al. [25] indicated that pyocyanin has several toxic effects on host cells, including depletion of NADH, glutathione, decomposing hydrogen peroxidase and other antioxidant in the host cell an alteration of the redox status and generation of oxygen radicals. Laxmi \& Bhat [26] reported that pyocyanin isolated from P. aeruginosa (BTRY1) were significant as higher radical scavenging activities at concentration very much lower than the ascorbic acid. 


\section{Anticancer Activity of Pyocyanin}

Hassani et al. [27] reported that the cytotoxicity of pyocyanin produced by both strains P. aeruginosa PHA-1 and mutant S300-8, against cancer cell line RD and normal cell line REF revealed that low concentrations (7.81-31.25 $\left.\mu \mathrm{g} \mathrm{ml}^{-1}\right)$ of had low activity on RD cell line with inhibition rate ranged from 28 to $36 \%$ after $24 \mathrm{hrs}$ of incubation. Also, it noticed that toxic efficiency of pyocyanin against RD cell line was increased at the highest concentrations (250 and $500 \mu \mathrm{g} \mathrm{ml}^{-1}$ ), the inhibition rate was $60 \%$ and $64 \%$, respectively after $72 \mathrm{hrs}$ of incubation. While, pyocyanin produced by mutant strain S300-8 was more efficient against growth of cancer cell line RD that produced by PHA-1. Pyocyanin inhibit growth of RD and achieved $65 \%$ of dead cells at low concentration, $7.81 \mu \mathrm{g} \mathrm{ml}^{-1}$, and notable an increase on growth inhibition of RD cell line $88 \%$ after $72 \mathrm{hrs}$ of incubation at pyocyanin concentration, $500 \mu \mathrm{g} \mathrm{ml}$ ${ }^{1}$. In contrast, pyocyanin either produced by PHA-1or S300-8 had no effectiveness on the viability of REF normal cells at all period of incubation.

Zhao et al. [28] revealed that pyocyanin had significantly inhibition against HePG2 cancer cells line proliferation and triggered the production of large amounts of reactive oxygen species (ROS), thereby up regulating superoxide dismutase (SOD) and catalase. In addition to depleted reduced glutathione (GSH) and decrease the GSH/oxidized GSH ratio. Muller et al. [29] reported that pyocyanin inhibited the proliferation of human dermal fibroblasts cell line. Also, Laxmi \& Bhat [26] indicated that pyocyanin isolated from $P$. aeruginosa (BTRY1) had reduced hemolytic activity even at concentration $1 \mathrm{\mu g} \mathrm{ml}^{-1}$. Also, reported that that the pyocyanin was not cytotoxic against normal cells line at concentrations ranged from 6.25 to $100 \mu \mathrm{g} \mathrm{ml}^{-1}$. It was observed that the cells showed almost $90 \%$ viability after pyocyanin treatment $6.25 \mathrm{\mu g} \mathrm{ml}^{-1}$. The cell viability of around $80 \%$ even at high concentrations indicating its safety of use in food consumption for human.

\section{Applications of Pyocyanin}

\section{Biological applications}

Phenazine compounds that produced by Pseudomonas spp. were known to possess a wide spectrum antimicrobial activity toward bacteria, fungi and eukaryotic cells. Also, several phenazine compounds showed antitumor, antimalaria and antiparasitic activities [30-31]. The antimicrobial activities of phenazine had been used in sustainable agriculture as a biocontrol agent against some food spoilage and pathogenic bacteria and fungi $[32,33]$. So, these compounds suitable to restrain microbes in agricultural and pharmaceutical application [34]. Phenazine was used to suppression of Erwinia amylovora which causes fire blight disease in apple flowers [35] and used as natural suppression of Fusarium wilt disease [36].

Pyocyanin was required for prevention of disease symptoms in plants and killing of nematode Caenohabditis elegans and the fruit fly Drosophila melanogaster $[37,38]$. Various plant roots colonizing Pseudomonas spp. had been shown to be potent microbiological control agents in various plant pathogen systems [39]. In 1986 found that phenazine compounds inhibit mycelia growth of several fungal pathogens of plants, phenazine produced by the root colonizing bacteria $P$. fluorescens and $P$. aureofaciens, had a dominant role in the control of take all disease of wheat caused by Gaeumannomyces graminis var. tritici $[40,41]$. phenazine had broad spectrum action against active and dormant structure of fungal phathogen Pythium aphanidermatum and root knot nematode Meloidogyne inconita. Phenazine derivatives were also used to reduce the chemical pesticides in agriculture which could be used either alone or in combination with pesticides to lower the doses of chemicals needed to obtain a profitable crop yield [42].

In medical applications, phenazine and their derivatives used in antifungal activity against variety of microorganisms e.g., Candida albicans and Aspergillus fumigatus, the risk factor of pulmonary candidiasis in patients [19]. Phenazine also known as tubermycin B because its antibiotic activity against Mycobacterium tuberculosis, which causing pneumonia and often fatal infections in susceptible patient population [43]. Also, Allen et al. [44] indicated that phenazine production leads to reduced chemokine and cytokine production by reduced neutrophil numbers and accelerated neutrophil apoptosis, which were associated with impaired bacterial clearance. Active proliferation of human lymphocytes was inhibited by pyocyanin [45]. The development of synthetic anticancer phenazine derivatives was an ongoing area of research aimed at combining known phenazine biological activities with increased target specificity towards cancer cells [46].

\section{Biotechnological Applications}

Microbial fuel cells (MFCs) use microorganisms to catalyze the conversion of chemical energy into electrical energy [47]. An ongoing issue with MFCs was that the slow rate of electron transfer from the microorganism to the anodic electrode limits MFC efficiency. Sanderson et al. [48] reported that phenazine methosulfate or phenazine ethosulfate served as good electron acceptors in photoelectrochemical cells. Rabaey et al. [49] revealed that addition of pyocyanin to MFC-containing Brevibacillus sp. PTH1 doubled the rate of electron transfer. The addition of a PCAproducing $P$. chlororaphis or a derivative that produces high levels of PCN to a mixed MFC also resulted in higher electron transfer rates [50].

Beneficial Pseudomonas species reach root surfaces chemotactically by flagella motility and colonise them. It was found that Pseudomonas also promotes the plant growth. The root and rhizosphere offer an ecological niche [51]. The interaction of $P$. aeruginosa with plants as a beneficial association was found to be quite common. P. aeruginosa, produces pyocyanin which is present in the rhizosphere soil and other sources. In soil it promotes direct plant growth and protects plants from the phytopathogens [5254]. Ali Siddiqui et al. [55] reported the use of rhizobacteria in the control of root rot and root knot disease complex of mungbean. The organism isolated from the rhizosphere was found to be P. aeruginosa which acted as a bio-control agent in inhibiting the growth of Macrophomina phaseolina, Fusarium solani and Rhizoctonia solani. Pseudomonas also showed nematicidal activity in killing the second stage larvae of Meloidogyne javanica.

The role of pyocyanin from P. aeruginosa 7NSK2 strain, in inducing resistance to Botrytis cinerea that causes infection in tomato and grapevine was demonstrated by Audenaert et al. [56]. Also, Anjaiah et al. [57] reported that pyocyanin from Pseudomonas species isolated from rhizosphere soil were used as biocontrol agent against Fusarium, the causative agent of wilt of chickpea and Pythium damping of bean. Sunish kumar et al. [58] revealed that pyocyanin produced by particular strains of $P$. aeruginosa strain PUPa3 showed biocontrol activity against a wide range of phytopathogenic fungi that infect rice, groundnut, tobacco, chilli, 
mango, sugarcane, tea, cotton and banana crops. The minimal inhibitory concentration of pyocyanin was found to be $29 \mu \mathrm{g} \mathrm{ml}^{-1}$ for Sclerotium rolfsii NCM1084, which also inhibited the growth of phytopathogens such as Aspergillus niger NCIM 1025, Fusarium oxysporum NCIM 1008, S. rolfsii NCIM1084 and Colletotricum falcatum [43].

De Vleesschauwer et al. [59] reported the use P. aeruginosa 7NSK2 strain as a biocontrol agent against the leaf blast (Magnaporthe grisea) and sheath blight ( $R$. solani) in the monocot model rice plant. $P$. aeruginosa 7NSK2 was treated in root which protected rice against leaf blast leaving unprotected from sheath blight. The pyocyanin produced by 7NSK2 enhanced the production of $\mathrm{H}_{2} \mathrm{O}_{2}$ in roots and leaves, which degraded the toxic enzyme from the plants. Onbasli \& Aslim [60] found that pyocyanin from Pseudomonas inhibits the E. coli isolates from sugar beet molasses. Pseudomonas strains isolated from the rhizosphere plant were used to treat against various species of Fusarium, Ralstonia and Meloidogyne which cause wilting disease in coleus and ashwagandha species [61]. The mechanism involved was studied and it was suggested that siderophore, HCN, indole acetic acid, pyocyanin and other volatile metabolites synthesized by Pseudomonas strain exhibits the biocontrol effect and they can also act as plant growth enhancers.

Khamdan \& Suprapta [62] found that P. aeruginosa act as very good biopesticides against soyabean stunt virus (SSV). $P$. aeruginosa has been isolated from rhizospheres of soyabean and formulated in various forms such as liquid formulation, together with polyacrylamide hydrogel. The formulations were effective in plant growth and increased resistance against SSV also increases the yield, chlorophyll content and peroxidase activity. The study concluded that the application of $P$. aeruginosa formulation was effective against SSV.

\section{CONCLUSION}

Pigments that produced as secondary metabolites to protect microorganisms from injurious effect of light rays of visible and near ultraviolet range had also several biological activities. Pyocyanin showed antibacterial and antifungal activity against several pathogenic bacteria and mycotoxigenic fungi also possess antioxidant and anticancer activity against cancer cell lines. These finding can be used as base for food industries as food preservation and in pharmaceuticals applications.

\section{REFERENCES}

1. Ran HM, Hassett DJ, Lau GW (2003) Human targets of Pseudomonas aeruginosa pyocyanin. Proc. Natl Acad Sci USA 100(24): 14315-14320.

2. Hassett, DJ, Charniga L, Bean K, Ohman DE, Cohen MS (1992) Response of Pseudomonas aeruginosa to pyocyanin: mechanisms of resistance, antioxidant defenses and demonstration of a manganese-cofactored superoxide dismutase. Infect Immun 60(2): 328-336.

3. Mavrodi DV, Bonsall RF, Delaney SM, Soule MJ, Phillips G, et al. (2001) Functional analysis of genes for biosynthesis of pyocyanin and phenazine-1-carboxamide from Pseudomonas aeruginosa PA01. J Bacteriol 183(12): 6454-6465.

4. Parsons JF, Greenhagen BT, Shi K, Calabrese K, Robinson H, et al. (2007) Structural and functional analysis of the pyocyanin biosynthetic protein Phz M from Pseudomonas aeruginosa. Biochemistry 46(7): 1821-1828.

5. Cox CD (1986) Role of pyocyanin in the acquisition of iron from transferring. Infect Immun 52(1): 263-270.

6. Hassan HM, Fridorich I (1980) Mechanism of the antibiotic action of pyocyanin. J Bacreriol 141(1): 156-163.
7. Baron SS, Rowe JJ (1981) Antibiotic action of pyocyanin. Antimicrobial Agents Chemother 20(6): 814-820.

8. Price Whelan A, Dietrich LE, Newman DK (2007) Pyocyanin alters redox homeostasis and carbon flux through central metabolic pathways in Pseudomonas aeruginosa PA14. Journal of Bacteriology 189(17): 63726381.

9. Baron SS, Terranova G, Rowe JJ (1989) Molecular mechanism of the antimicrobial action of pyocyanin. Curr Microbiol 18(4): 223-230.

10. Vukomanovic DV, Zoutman DE, Stone JA, Marks GS, Brien JF, et al. (1997) Electrospray mass-spectrometric, spectrophotometric and electrochemical methods do not provide evidence for the binding of nitric oxide by pyocyanine at pH 7. Biochemistry Journal 322(1): 25-29.

11. Karpagam S, Sudhakar T, Lakshmipathy M (2013) Microbicidal response of pyocyanin produced by P. Aeruginosa toward clinical isolates of fungi. Int J Pharm Pharm Sci 5(3): 870-873.

12. Audenaert K, Pattery T, Cornelis P, Hofte M (2002) Induction of systemic resistance to Botrytis Cinerea in tomato by Pseudomonas aeruginosa 7NSK2: role of salicylic acid, pyochelin and pyocyanin. Mol Plant Microbe Interact 15(11): 1147-1156.

13. Saha S, Thavasi R, Jayalakshmi S (2008) Phenazine pigments from Pseudomonas aeruginosa and their application as antibacterial agent and food colourants. Res J Microbiol 3(3): 122-128.

14. Fontoura R, Spada JC, Silveira ST, Tsai SM, Brandelli A (2009) Purification and characterization of an antimicrobial peptide produced by Pseudomonas sp. strain 4B. World J Microbiol Biotechnol 25: 205-213.

15. Rahman PK, Pasirayi G, Auger V, Ali Z (2009) Development of simple and low-cost micro-bioreactor for high throughput bioprocessing. Biotechnol Lett 31(2): 209-214.

16. Sweden EG (2010) Study the effect of antibiotics on pyocyanin production from Pseudomonas aeruginosa and pyocyanin as antibiotic against different pathogenic bacteria. J Univ Anbar Pure Sci 4(1): 15-18.

17. Sudhakar T, Karpagam S, Shiyama S (2013) Antifungal efficacy of pyocyanin produced from bioindicators of nosocomial hazards. Int J ChemTech Res 5: 1101-1106.

18. Kerr JR (1994) Suppression of fungal growth exhibited by Pseudomonas aeruginosa. J Clin Microbiol 32(2): 525-527.

19. Kerr JR, Taylor GW, Rutman A, Hoiby N, Cole PJ, Wilson R (1999) Pseudomonas aeruginosa pyocyanin and 1-hydroxyphenazine inhibit fungal growth. J Clin Pathol 52(5): 385-387.

20.Pal R, Revathi R (1998) Susceptibility of yeasts to Pseudomonas aeruginosa. Indian J Med Microbiol 16(2): 72-74.

21. Kaleli I, Cevahir N, Demir M, Yildrim U, Sahin R (2007) Anticandidal activity of Pseudomonas aeruginosa strains isolated from clinical specimens. Mycoses 50(1): 74-78.

22. Hogan DA, Kolter R (2002) Pseudomonas-Candida interaction: an ecological role for virulence factors. Science 296(5576): 2229-2232.

23. Gibson J, Sood A, Hogan DA (2009) Pseudomonas aeruginosa-Candida albicans interactions: localization and fungal toxicity of a phenazine derivatives. App Environ Microbiol 75(2): 504-513.

24. Hassanein WA, Awny NM, El-Mougith AA, Salah El-Dein SH (2009) Characterization and antagonistic activities of metabolite produced by Pseudomonas aeruginosa Sha8. J Appl Sci Res 5(4): 392-403.

25. Rada B, Jendrysik MA, Pang L, Hayes CP, Yoo Dg, et al. (2013) Pyocyanin enhanced neutrophil extracellular trap formation requires the NADPH oxidase. PLoS One 8(1): e54205.

26. Laxmi MS, Bhat G (2016) Characterization of pyocyanin with radical scavenging and antibiofilm properties isolated from Pseudomonas aeruginosa strain BTRY 1. 3 Biotech 6(1): 27.

27. Hassani HH, Hasan HM, Al-Saadi A, Ali AM, Muhammad MH (2012) A comparative study on cytotoxicity and apoptotic activity of pyocyanin produced by wild type and mutant strains of Pseudomonas aeruginosa. Eur J Exp Biol 2(5): 1389-1394. 
28. Zhao J, Wu Y, Alfred AT, Wei P, Yang S (2014) Anticancer effects of pyocyanin on HepG2 human hepatoma cells. Letters in applied microbiology 58(6): 541-548.

29. Muller M, Li Z, Maitz PK (2009) Pseudomonas pyocyanin inhibits wound repair by inducing premature cellular senescence: role for $\mathrm{p} 38$ mitogenactivated protein kinase. Burns 35(4): 500-508.

30. Laursen J, Nielsen J (2004) Phenazine natural products: Biosynthesis, synthetic analogue and biological activity. Chem Rev 104(3): 1663-1685.

31. Brisbane P, Janik L, Tate M, Warren R (1987) Revised structure for the phenazine antibiotic from P. fluorescens 2-79. Antimicrob Agents Chemo 31(12): 1967-1971.

32. Kim K (2000) Phenazine-1-carboxylic acid resistance in phenazine-1carboxilic acid producing Bacillus sp. B-6 J Biochem. Mol Biol 33: 332 336.

33. Aunchaleeb N, Sukanga A, Chanokporn P, Paweena P, Saksit C, et al. (2009) Synthesis, isolation of phenazine derivatives and their antimicrobial activity. Walailak J Sci and Tech 6(1): 79-91.

34. Giddens S, Houliston G, Mahanty H (2003) The influence of antibiotic production and pre-emptive colonization on the population dynamics of Pantoea agglomerans (Erwinia herbicola) Eh1087 and Erwinia amylovora in planta. Environ Microbiol 5(10): 1016-1021.

35. Mazurier S, Corberand T, Lemanceau P, Raaijmarkers J (2009) Phenazine antibiotics produced by fluorescent pseudomonads contribute to natural suppressiveness to Fusarium wilt. ISME J 3(8): 977-991.

36. Mahajan M, Tan M, Rahme L, Ausubel S (1999) Molecular mechanisms of bacterial virulence elucidated using a Paeruginosa- Genorhabditis elegans phathogenesis model. Cell 96(1): 47-56.

37. Rahme L, Ausubel F, Cao H, Drenkard E, Goumnerov B, et al. (2000) Plants and animals share functionally common bacterial virulence factors. Proc Natl Acad Sci USA 97: 8815-8821.

38. Thomashow L, Weller D (1995) Current concepts in the use of introduced bacteria for biological disease control: Mechanisms and antifungal metabolites. Stacey G, Keen N eds. Plant-Microbe Interations. Chapman and Hall, New York, USA Pp:187-235.

39. Hamdan H, Weller D, Thomashow L (1991) Relative importance of fluorescent siderophores and other factors in biological control of Gaeumannomyces gramins var. tritci by $P$ fluorescens 2-79 and M4-80R. Appl Environ Microbiol 57: 3270-3277.

40. Pierson L, Thomashow L (1992) Cloning and heterologous expression of the phenazine biosynthetic locus from $P$ aureofaciens $30-84$. Mol Plant Microbe Interact 5(4): 330-339.

41. Chin AWT, Bloemberg G, Lugtenberg B (2003) Phenazines and their role in biocontrol by Pseudomonas bacteria. New Phytol 157(3): 503-523.

42. Rane MR, Sarode PD, Chaudhari BL, Chincholkar SB (2007) Detection, isolation and identification of phenazine 1-carboxylic acid produced by biocontrol strains of Pseudomonas aeruiginosa. J Sci Ind Res 66: 627-631.

43. Allen L, Dockrell D, Pattery T, Lee D, Cornelis P, et al. (2005) Pyocyanin production by $P$ aeruginosa induce neutrophil apoptosis and impairs neutrophil-mediated-host defenses in vivo. J immunology 174(6): 36433649.

44. Sorensen R, Klinger J, Cash H, Chase P, Dearborn D (1983) In vitro inhibition of lymphocyte proliferation by $P$. aeruginosa PAo1. Infection and Immunity 4: 1673-1682.
45. Nakaike S, Yamagishi T, Nanaumi K, Otomo S, Tsukagoshi S (2005) CellKilling activity and kinetic analysis of a noval antitumor compound NC190, a Benzo $[\alpha]$ phenazine derivative. Jpn J Cancer Res 83(4): 402-409.

46. Hari N, Moorthy N, Karthikeyan C, Trivedi P (2009) Endogenous nitric oxide protects bacteria against a wide spectrum of antibiotics. Science 325(5946): 1380-1384

47. Torres C, Marcus A, Lee HPP, Krajmalnik B, Rittmann B (2010) A kinetic perspective on extracellular electron transfer by anode-respiring bacteria. FEMS Microbiol Rev 34(1): 3-17.

48. Sanderon D, Gross E, Seibert M (1987) A photosynthetic photoelectrochemical cell using phenazine methosulfate and phenazine ethosulfate as electron acceptors. Appl Biochem Biotechnol 14: 1-12.

49. Rabaey K, Boon N, Hofte M, Verstraete W (2005) Microbial phenazine production enhances electron transfer in biofuel cells. Environ Sci Technol 39(9): 3401-3408.

50. Bais HP, Weir TL, Perry LG, Gilory S, Vivanco JM (2006) The role of root exudates in rhizosphere Interactions with plants and other organisms. Annu Rev Plant Biol 57: 233-236.

51. Cook JR (1988) Biological control and holistic plant health care in agriculture. Am J Altern Agric 3(2-3): 51-62.

52. Glick BR (1995) The enhancement of plant growth of free-living bacteria. Can J Microbiol 41(2): 109-117.

53. Mercado BJ, Bakker PA (2007) Interactions between plants and beneficial Pseudomonas sp.: exploiting beneficial traits for crop protection. Antonie Van Leeuwenhoek 92(4): 367-389.

54. Ali SI, Ehetshamul HS, Shahid SS (2001) Use of rhizobacteria in the control of root rot-root knot disease complex of mungbean. J Phytopathol 149(6): 337-346.

55. Audenaert K, Pattery T, Cornelis P, Hofte M (2002) Induction of systemic resistance to Botrytis Cinerea in tomato by Pseudomonas aeruginosa 7NSK2: role of salicylic acid, pyochelin and pyocyanin. Mol Plant Microbe Interact 15(11): 1147-1156.

56. Anjaiah V, Cornelis P, Koedam N (2003) Effect of genotype and root colonization in biological control of Fusarium wilts in pigeon pea and chickpea by Pseudomonas aeruginos PNAI. Can J Microbiol 49(2): 85-91.

57. Sunish KR, Ayyadurai N, Pandiaraja P, Reddy AV, Venkateswarlu Y, et al. (2004) Characterization of anti-fungal metabolite produced by a new strain Pseudomonas aeruginosa PUPa3 that exhibits broad spectrum antifungal activity and biofertilizing traits. J Appl Microbiol 98(1): 145154

58. De Vleesschauwer D, Cornelis P, Hofte M (2006) Redox active pyocyanin secreted by $P$ aeruginosa 7 NSK2 triggers systems resistance to Marnaporthe grisea but enhances Rhizoctoria solani susceptibility in rice. Mol Plant Microbe Interact 19(12): 1406-1419.

59. Onbasli D, Aslim B (2008) Determination of antimicrobial activity and production of some metabolites by pseudomanas aeruginosa B1 and B2 in sugar beet molasses. Afr J Biotechnol 7(24): 4614-4619.

60. Mallesh SB (2008) Plant growth promoting rhizobactaria their characterization and mechanism in the suppression of soil borne pathogens of coleus and ashwaghandha. PhD thesis, University of Agricultural Sciences, Dharwad, India.

61. Khamdan K, Suprapta DN (2011) Induction of plant resistance against soyabean stunt virus using some formulations of Pseudomonas aeruginosa. J ASSAAS 17: 98-105. 